\title{
Pour une recherche technologique en sciences humaines et sociales
}

\author{
Charles Lenay $^{1, \mathrm{a}}$, Pascal Salembier ${ }^{2, \mathrm{~b}}$, Pierre Lamard ${ }^{3, \mathrm{c}}$, Yves-Claude Lequin ${ }^{3, \mathrm{c}}$ \\ et Loïc Sauvee ${ }^{4, d}$ \\ ${ }^{1}$ COSTECH-UTC, France \\ ${ }^{2}$ TechCICO-UTT, France \\ ${ }^{3}$ RECITS-UTBM, France \\ ${ }^{4}$ PICAR-T- LaSalle Beauvais, France
}

\begin{abstract}
Résumé. Nous proposons ici une approche originale de la technologie dans les sciences humaines et sociale (SHS), approche qui sera développée dans le cadre d'un groupement d'intérêt scientifique (GIS) pour l'unité des technologies et des sciences de l'homme. En effet, la place des SHS dans l'organisation de la recherche et des enseignements dispensés dans les écoles d'ingénieurs pose question. Les attentes vis-à-vis des enseignants-chercheurs sont souvent l'objet de malentendus et renvoient pour partie à une méconnaissance réciproque entre SHS et sciences pour l'ingénieur (SPI) mais aussi, plus fondamentalement à une coupure entre enseignement et recherche. Ces obstacles illustrent également la difficulté à mettre en place une recherche interdisciplinaire pérenne qui associe SHS et SPI. Nous proposons ici une initiative qui vise à fonder une recherche interdisciplinaire de ce type sur la question de la « constitutivité mutuelle » de l'Homme et de la technique.
\end{abstract}

\begin{abstract}
We propose an original approach to technology in the humanities and social sciences (HSS), an approach that will be developed as part of a scientific interest group (SIG) for the unity of technologies and human sciences. Indeed, the place of the HSS in the organization of research and education given in engineering schools and technical universities raises many questions. Expectations related to this training are very often a source of misunderstandings, due to a mutual ignorance between HSS and engineering sciences (ES), but also to a separation between research and education. These issues illustrate as well the difficulties encountered by the general project of an interdisciplinary research associating HSS and ES. We propose here an initiative which aims at building an interdisciplinary project grounded on the question of the relationship of "mutual shaping" between human and technology.
\end{abstract}

\footnotetext{
a e-mail : charles.lenay@utc.fr

b e-mail : Pascal.Salembier@utt.fr

c e-mail : pierre.lamard@utbm.fr; yves.lequin@utbm.fr

d e-mail : Loic.Sauvee@lasalle-beauvais.fr
}

This is an Open Access article distributed under the terms of the Creative Commons Attribution License 4.0, which permits unrestricted use, distribution, and reproduction in any medium, provided the original work is properly cited. 


\section{Introduction}

Les éternels discours pour dépasser l'opposition des deux cultures, entre sciences de l'homme et sciences de la nature (sciences de la matière, sciences de la vie), semblent toujours buter sur de mêmes écueils : partages de territoires, disputes aux frontières, indifférence ou rapports hiérarchiques d'instrumentalisation réciproque, définition des objets (Akrich, 1987). Avant d'y revenir, notons que ces questions sont particulièrement sensibles dans le contexte des centres technologiques comme les écoles d'ingénieurs où l'enseignement en sciences humaines et sociales est souvent soumis à une série d'injonctions dont nous verrons qu'elles sont illusoires comme « adapter les ingénieurs à l'environnement socio-économique »; ou « favoriser l'acceptabilité sociale du changement technique » (Jeneveau, 2011). Nous voulons ici proposer les principes et méthodes que nous avons élaborés pour dépasser ces difficultés. Nous verrons que de telles incompréhensions résultent d'une coupure entre enseignement et recherche, coupure particulièrement significative autour de la question technique parce que l'approche qui depuis deux siècles préside aux formations techniques françaises consiste à réduire les techniques à des sciences physiques appliquées.

Pourtant, l'enseignement et la recherche en sciences humaines et sociales dans les universités de technologie françaises se sont développées d'une façon originale et ont progressivement élaboré quelques solutions à ces difficultés. Depuis les premières propositions de Guy Denielou (présidentfondateur de l'université de technologie de Compiègne en 1972) pour un " Ingénieur philosophe » (Lamard \& Lequin, 2005) ces écoles ont intégré 20 à $30 \%$ d'enseignements en SHS dans la formation de leurs ingénieurs, et ont progressivement créé des équipes de recherche associées en SHS ou des équipes de recherche pluridisciplinaires comportant une composante SHS forte (Costech à l'UTC, TechCICO et le CREIDD à l'UTT, Récits à l'UTBM).

Alors que ces équipes, également associées à l'équipe PICAR-T de LaSalle Beauvais, sont engagées dans un processus de rassemblement au sein d'un Groupement d'Intérêt Scientifique UTSH (Unité des technologies et sciences de l'homme), elles ont proposé quelques éléments réflexifs sur la démarche originale en SHS qui s'est peu à peu dégagée de ce contexte technologique.

Nous voulons proposer ici quelques uns des principes directeurs de cette recherche technologique en sciences humaines. Pour cela il est d'abord nécessaire de ressaisir le mouvement historique qui a présidé à cette rencontre originale entre SHS et sciences de l'ingénieur (SPI).

\section{Des humanités techniques jusqu'à nos jours}

A la Libération et dans le contexte du plan Langevin-Wallon, le gouvernement veut poursuivre l'unification du système public d'enseignement (« Ecole unique»). Considérant que « si la profession isole, la culture doit rapprocher (Mialaret, 1997) $^{1}$, dans le domaine de l'enseignement technique, il est prévu entre autres pour les élèves un enseignement général spécifique, sous le nom d' « humanités techniques » (Bonnet \& Terral, 2011). Cette philosophie est portée par P. Le Rolland, Directeur de l'Enseignement technique, avec l'appui de G. Friedmann, sociologue du travail, Gilbert Simondon, philosophe, L. Febvre, historien. Le lycée comptait des « humanités classiques » et des « humanités scientifiques », l'idée est d'établir des « humanités techniques » (plus tard nommées « culture technique »), afin de rapprocher les trois filières (littéraires, scientifiques et techniques), les enseignants d'ENNA formant les futurs enseignants des écoles d'apprentissages devenant collèges d'enseignement technique après 1959, sont formés à une nouvelle pédagogie, dont la mise en œuvre s'avèrera difficile,

\footnotetext{
${ }^{1}$ Plan Langevin-Wallon - texte présenté par Mialaret Gaston. pp 33 et 41. Le regroupement dans les universités est prévu grâce à des « instituts techniques reliés aux facultés et aux centres d'études », ainsi qu'une formation pédagogique pour les intervenants extérieurs des «enseignements spéciaux»: "Les enseignements spéciaux sont ceux pour lesquels l'université doit recruter des maîtres qui aient l'expérience de l'industrie, de l'agriculture, des affaires, de l'administration, bref de la formation sous toutes ses formes et à tous ses niveaux » (Mialaret, 1997, p 41).
} 
beaucoup d'enseignants trouvant cette pédagogie trop intellectuelle, et de nombreux élèves « boudant » ces enseignements généraux (Bonnet \& Terral, 2011). En définitive, dans le secondaire - jusqu'à nos jours - l'enseignement professionnel et technique (même lorsqu'il est rebaptisé « technologique ») demeure - pour l'essentiel - dépourvu d'enseignements en sciences humaines et sociale, surtout lorsqu'il s'agit de comprendre les processus techniques. (Lequin, 2009 ; Charmasson, 1987 ; Bodé, 2002) $)^{2}$. Herbert Simon évoque même un recul ${ }^{3}$.

Cependant, vers 1945-1950, dans l'enseignement technique supérieur, on dénombre 82 écoles d'ingénieurs, majoritairement privées et étroitement spécialisées, avec un essor des ENSI (écoles nationales supérieures d'ingénieurs, créées par le décret du 16 janvier 1947) ${ }^{4}$. Dans l'ensemble la formation des ingénieurs est assurée dans l'esprit d'une « technonomie » (ou « logique de la technique »), à forte teneur en mathématique, physique ou mécanique, mais sans formations générales (qui sont dispensées par les universités) (Prost, 1997). Au milieu des années 1950, les sciences sociales (re)viennent à l'ordre du jour, à la fois pour des raisons scientifiques et pour des raisons très pragmatiques, de gestion des entreprises et de la société (Bernard, 1970 ; Musselin, 2001) ${ }^{5}$. Prenons l'exemple du CUCES (Centre universitaire de coopération économique et sociale) de Nancy (Laot, 1999) qui assure depuis 1954 une formation économique et sociale des élèves ingénieurs. En juin 1957, dans un rapport pour le IIIe Plan, sur « Recherches et progrès technique », H. Longchambon souligne les besoins nationaux en recherche technique et aussi en sciences sociales ${ }^{6}$.

C'est dans ce contexte que les initiateurs de l'INSA de Lyon (Longchambon, Billères, G. Berger et Capelle) conçoivent un « département des Humanités ». C'est une première en école d'ingénieurs (Lequin, 2013). Inaugurés respectivement par A. Sauvy et F. Perroux, un bâtiment et une rotonde sont réservés spécialement aux enseignements et aux conférences des Humanités. Dans la pratique, il s'agit d'initier concrètement les futurs ingénieurs « aux problèmes humains des entreprises », objectif indispensable, réclamé depuis nombre d'années, mais assez éloigné des recherches académiques en sciences humaines et sociales (Boirel, 1983). Cette « innovation » sera mainte fois contrainte, sous la pression notamment des départements de génie ; le département des Humanités sera réduit à un Centre, lorsque ces derniers deviennent diplômants ${ }^{7}$. Actuellement, le principe même d'un enseignement par la recherche (c'est-à-dire un enseignement qui s'appuie sur une recherche en cours et qui donne à comprendre les pratiques de cette recherche $)^{8}$ est remis en cause, privilégiant les enseignements « classiques » des langues étrangères et des enseignements SHS, directement professionnalisant.

\footnotetext{
${ }^{2}$ Voir aussi (Charlot \& Figeat, 1985). Selon Léon Antoine (Léon, 1968, p. 100) : «L'enseignement technique élémentaire, tel qu'il est régi par la loi Astier, consiste moins en une formation en école qu'en un aménagement de la formation sur le tas. ». Le texte que Marc Bloch publia en 1943 («Notes pour une révolution de l'enseignement») est reproduit in (Bloch, 1957) pp. 254268. Les références sont de cette réédition. Pour saisir le contexte (Becker \& Bloch, 2006) : « L'enseignement secondaire a pour objet de former des esprits; non, par avance, des ingénieurs, des chimistes ou des arpenteurs. Ceux-là trouveront plus tard et ailleurs, les écoles qu'il leur faut » (p. 267).

3 «Par un paradoxe ironique, alors que s'affirme le rôle décisif de la conception dans toute activité professionnelle, les sciences naturelles au XXe siècle, surtout dans les deux ou trois décennies qui ont suivi la Seconde Guerre mondiale, ont presque complètement éliminé les sciences de l'artificiel du programme des écoles formant des professionnels. Les écoles d'ingénieurs sont devenues des écoles de physique et de mathématiques (...)» (Simon, H. A. (1969), p. 203 dans sa version française (Gallimard 2004).

${ }^{4}$ Pour une part héritières des anciens instituts associés (depuis 1883) aux facultés des sciences, ces écoles d'ingénieurs sont incluses dans les universités (André Grelon).

5 « Le technologue, à la différence du scientifique, sera un homme de synthèse dont l'ambition doit être de construire quelque chose de nouveau, utile à la société. (...). Cela suppose que le technologue supérieur ait une formation sociale et humaine importante qui lui permette de rassembler autour de lui une équipe de spécialistes. » (Bernard, 1970, pp 19-20).

${ }^{6}$ Fernand Braudel a rédigé cette partie («Les Sciences sociales en France. Un bilan, un programme »), et la publie sous la signature de Henri Longchambon (Longchambon, 1958).

${ }^{7}$ Le centre n'est pas une structure institutionnelle reconnue en tant que telle au sein de l'établissement et ne peut pas prétendre à une quelconque autonomie ni dans l'offre de formation, ni dans les activités de recherche.

${ }^{8}$ Il n'y a plus de structure de recherche « estampillé » clairement SHS qui puissent justifier un enseignement par la recherche.
} 
Cependant, un laboratoire de recherche en sciences sociales voit le jour en 2004 : STOICA (Savoirs, Techniques, Organisation, Innovation, Conception Appliqués), devenu ITUS en 2013 (Ingénieries, Techniques, Urbanisations, Sociétés) (Lequin \& Lamard, 2011) mais perdant sa lisibilité strictement SHS.

Nous ne pouvons pas mener ici une analyse détaillée des raisons conjoncturelles et structurelles, politiques et épistémologiques de ces échecs relatifs. Notons seulement qu'ils ont souvent résulté de conflits dans lesquels les facultés universitaires revendiquaient une autonomie qu'elles sentaient menacée par l'utilitarisme technologique, par le risque d'une instrumentalisation de leur savoir qui serait au service de technologies développées préalablement suivant l'application aveugle des résultats des sciences physiques. Le développement universitaire des SHS semble s'être fait dans une relative défiance (même si parfois elle était mêlée d'envie et d'imitation) vis-à-vis des autres sciences de la nature. Cette séparation disciplinaire et institutionnelle a perduré dans la majorité des universités et centres de recherches français. ${ }^{9}$

Parallèlement aux avatars de l'INSA, un nouveau projet est évoqué par des cercles restreints issu du 2e colloque de Caen en 1966. La conception d'un profil universitaire spécifique est porté par divers mouvements de mai-juin 1968, puis par plusieurs partis au Parlement. Au sein du cabinet E. Faure un projet d'universités techniques est préparé, dont la première implantation aurait dû être localisée à Villetaneuse (Lamard \& Lequin, 2012a). Ce projet sera entravé en 1969, après la démission de Charles de Gaulle puis par l'élection de G. Pompidou à la présidence de la République. Olivier Guichard, nouveau ministre de l'Education nationale établit un autre groupe de travail, avec pour objectif de fonder une troisième voie universitaire, entre grandes Ecoles techniques et universités, mais aussi entre grandes Ecoles et établissements formant exclusivement aux méthodes industrielles (Arts et Métiers ou Ecole Nationale d'Ingénieurs). C'est ce groupe de travail (piloté par Bernard Delapalme ${ }^{10}$ ) qui, en deux ans, conçoit l'université de technologie de Compiègne (UTC), ouverte en septembre 1972, imaginée comme première pierre d'un réseau à généraliser. Après 1981, des efforts sont multipliés pour créer d'autres Universités de technologie, mais pour quinze essais, deux autres seulement verront le jour : à SevenansBelfort en 1985 (qui deviendra UTBM en 1999, après fusion avec l'ENI locale), puis à Troyes en 1992.

Une des originalités de l'UTC est de prévoir un département Technologie et Sciences de l'Homme (TSH), qui -tirant des leçons de l'INSA de Lyon (Lamard \& Lequin, 2005) - est prévu puissant et diplômant : pour devenir ingénieur chaque étudiant doit obtenir un tiers d'unités de valeur en sciences humaines et sociales; et majoritairement, les enseignants font partie de l'UT, sans dépendre d'une autre université. Les sciences humaines et sociales qui sont enseignées couvrent un vaste champ (sociologie, histoire, économie, droit, philosophie, langues, etc.) sans pour autant être calquées sur le programme d'une faculté des lettres et sciences humaines. Un département du même type sera créé à l'UTT et à l'UTBM en 1995 sous le nom de département « Humanités ». Des laboratoires voient le jour, menant à bien de vastes programmes de recherches scientifiques en sciences humaines et sociales et mobilisant des méthodes interdisciplinaires autour de l'objet technique ou de la technologie (Lamard \& Lequin, 2012b, 2012c). Au fil des années ces environnements concrets ont permis le développement d'un style intellectuel original et finalement à une démarche générale de recherche que nous voulons présenter ici.

\section{L’interdisciplinarité : un projet toujours contrarié}

A partir de l'expérience acquise dans ces écoles d'ingénieur, notre ambition est de proposer une recherche philosophique et scientifique qui prend les outils et systèmes techniques pour des faits

\footnotetext{
9 «Beaucoup de ceux qui sont restés fidèles à la conception pure de l'enseignement supérieur s'inquiètent de ce voisinage : c'est l'utilitarisme qui s'installe à côté de la culture désintéressée, c'est l'usine qui s'élève au-dessus du Parthénon et menace de l'écraser », Janet Paul, Notes et souvenirs, Gauthiers-Villars, Paris, 1923, p. 16. Cité par Grelon André, «Les universités et la formation des ingénieurs en France (1870-1914) », dans Formation emploi, 27-28, juillet-décembre 1989, p. 84.

10 Bernard Delapalme est directeur de la recherche scientifique et technique du groupe ELF-ERAP.
} 
humains, à la fois constitués et constituants, et non pas de simples applications conjoncturelles de connaissances physiques. Ce qui est souvent oublié ce n'est pas tant que les techniques soient produites par un contexte social, économique et idéologique, mais plutôt que les dispositifs concrets produits aient un sens, des valeurs et des effets cognitifs pour les activités sociales humaines. En effet, la question de la technique est partagée par les sciences de l'homme comme par les sciences de la nature.

D'une part, la technique est un fait anthropologique originaire et universel (Leroi-Gourhan, 1964 ; Sigaut, 2002, 2010). Entendons ici « technique » comme le système des objets, dispositifs et pratiques, qui est produit par l'activité humaine, qui sert à cette activité et qui est toujours déjà là, reçu de la collectivité, transformé et transmis. Ainsi la question technique est présente dans l'ensemble des sciences humaines et sociales, y compris la philosophie ou l'histoire .(Schlanger, 2012). L'histoire se fonde à partir des techniques de l'écriture et l'analyse des traces matérielles ; la sociologie décrit ses objets à partir des techniques du corps, des symboles concrets et des structures matérielles (Mauss, 1970 ; Haudricourt, 1988, 2011) ${ }^{11}$; la linguistique se développe à partir du processus de grammatisation décrit par Sylvain Auroux, etc. (Auroux, 1994 ; Garçon, 2012)

D'autre part, les sciences de la nature, qu'elles soient biologiques, physiques, ou mathématiques, sont liées aux techniques par leurs moyens comme par leurs conséquences revendiquées. Ce que l'on peut appeler la technoscience, correspond à la dynamique contemporaine de la recherche où les méthodes de l'expérimentation comme les modes de validation passent par la réalisation de dispositifs technologiques (Sebbah, 2010). Et a fortiori, dans le cas des « Sciences Pour l'Ingénieur» (SPI) les sciences de la nature sont mobilisées dans des disciplines technologiques (mécanique, informatique, électronique, automatique, etc.) qui travaillent directement sur les contraintes et les possibles du faire technique.

Pourtant, en dépit de cet objet partagé, les projets d'interdisciplinarité sans cesse annoncées échouent souvent à mettre en œuvre un réel travail commun (Schmid, Mambrini-Doudet, \& Hatchuel, 2011). Les deux écueils, biens connus mais toujours renouvelés sont, d'une part, une opposition entre culture et technique et d'autre part, un rôle purement instrumental et subalterne de la technique, ou réciproquement l'instrumentalisation des sciences humaines au service des développements industriels (Akrich, 1994). Une des raisons de cette situation vient sans doute du fait que, comme le souligne François Sigaut, la technique tient de l'implicite, et que son explicitation est difficile (Sigaut, 2010). L'urgence d'une prise en compte du fait technique par les SHS a été d'ailleurs souvent été mise en avant (Cresswell, 2011 ; Lemonnier, 1983 ; Mitcham, 1994).

L'opposition : la technique, considérée comme moyen, est opposée à la culture comprise comme source de sens ; le rapport matériel concret à la nature contre le discours social des valeurs ou des émotions. Chacun à sa place dans, au mieux, une coexistence polie.

L'instrumentalisation réciproque, soit des sciences humaines par les ingénieurs et techniciens, soit des techniques par les sciences humaines.

Dans le premier cas, les « humanités » sont seulement convoquées comme supplément d'âme ou comme vernis esthétique apposé aux dispositifs techniques pour favoriser leur acceptation individuelle

\footnotetext{
11 Haudricourt a inséré p. 37 du recueil de ses articles, publié par la MSH en 1988, une petite note précédant la reproduction de son article de 1964 «La technologie, science humaine»:

"Je me rappelle qu'en 1946, lors du projet d'Encyclopédie de la Renaissance, sous la direction de Henri Mougin, l'existence de la technologie en tant que discipline autonome me fut contestée, en particulier par Jean Bruhat. Mais à la même époque, Georges Friedmann me demanda un volume sur L'Homme et les techniques pour une collection qu'il dirigeait chez Gallimard. Lorsqu'Igor de Garine m'eut préparé illustrations et manuscrit, celui-ci fut refusé par Edgar Morin, qui avait succédé à Friedmann comme directeur de la collection, pour cause d'insuffisance sociologique. Quelques années plus tard, Jean Poirier me demanda de rédiger le chapitre "Technologie » dans son Ethnologie générale (Encyclopédie de la Pléiade), et je pus utiliser les illustrations et le manuscrit préparés avant 1960 pour ce chapitre, "La technologie culturelle, essai de méthodologie », qui ne parut qu'en 1968, accompagné d'un contre-article que Poirier, peu satisfait du mien, avait demandé à Jean Michéa ("La technologie culturelle, essai de systématique).

L'article suivant, bien que publié en 1964, fut donc rédigé après le chapitre de La Pléiade, publié en 1968. »
} 


\section{SHS Web of Conferences}

et sociale, ou bien, au mieux, il s'agit de proposer des aménagements techniques par la prise en compte de l'utilisateur. Dans le second cas, les sciences humaines s'emparent cette fois des techniques, mais seulement pour utiliser des terrains et des instruments supposés neutres relativement à leur propre questionnement.

Dans tous les cas, il n'y a pas de véritable dialogue interdisciplinaire, puisque les apports et questions ne viennent pas affecter des problématiques internes à chaque discipline, mais relèvent simplement de l'application de savoirs que l'on admet être acquis. Il y a une extériorité et une opacité des techniques pour les SHS, comme il y a une extériorité et une opacité des SHS pour les technologues. Cela flatte certainement le sentiment d'expertise et le souci d'indépendance de chaque champ disciplinaire, mais en même temps cela conduit à admettre sans critique diverses formes d'essentialisme ou de substantialisme pour les savoirs importés, ce qui est particulièrement trompeur pour le domaine essentiellement changeant de la technique et des usages. Ni la connaissance des contraintes techniques, ni celle des pratiques humaines, ne sont des choses fixes, stabilisées, mais seulement des objets pour une recherche toujours en cours.

Ainsi, au moment de concevoir une interface, tel designer prendra les résultats des neurosciences comme des acquis indiscutables ; au moment d'étudier les dynamiques de déplacement en ville, tel sociologue prendra comme stabilisées les techniques de géolocalisation ; au moment de réaliser son programme de visualisation en réalité virtuelle, tel informaticien tiendra pour prédéfinies les attentes perceptives des utilisateurs...

Séparation et instrumentalisation débouchent sur un apport faible, généralement inefficace, des sciences humaines pour le travail interdisciplinaire cependant nécessaire. Outre le désintérêt des chercheurs qui peuvent y craindre une perte d'autonomie, il y a là un risque de dogmatisme. En effet, à chaque fois que l'on admet un ensemble de connaissances comme stable et définitif on quitte la dynamique de la recherche scientifique. Ce dogmatisme scientifique, que ce soit dans les sciences de la nature ou dans les sciences humaines, ne permet ni de comprendre le monde contemporain et ses transformations, ni de pouvoir participer à de véritables processus d'innovation, ni d'ailleurs d'assurer un enseignement susceptible de préparer les étudiants aux mutations futures des techniques et des sciences.

\section{La technique : un objet de recherche pour les SHS}

Pour sortir de ces apories, non par un simple volontarisme institutionnel, mais de l'intérieur même de la dynamique des disciplines scientifiques et philosophiques, on peut faire de la constitutivité technique de l'expérience humaine l'objet même d'une recherche fondamentale en SHS. Il s'agit alors de travailler sur la co-construction entre humain et technique, à la fois comme constitutivité technique des pratiques, des activités cognitives et du social, et comme construction sociale des techniques et de leurs usages. Toutes les activités humaines sont « artificielles », c'est-à-dire portées par les outils et milieux techniques que nous recevons et modifions. L'objectif général de cette recherche est de comprendre comment chaque outil, chaque médiation ou dispositif technique, transforme nos façons de percevoir, de penser, de mémoriser, d'interagir et de construire du sens partagé (Steiner, 2010). Ceci peut mobiliser à chaque fois, non seulement la psychologie, l'ergonomie, l'anthropologie, la sociologie, l'histoire et la philosophie, les sciences économiques et de gestion, mais aussi le savoir technique lui-même, qui porte ses propres contraintes et ses propres questions. L'originalité d'une recherche sur la constitutivité technique de l'expérience humaine milite donc en faveur d'une nouvelle forme d'interdisciplinarité en sciences humaines et sociales, interdisciplinarité associant les sciences de l'ingénieur. Un tel travail interdisciplinaire permet d'appréhender des logiques de co-constitution qui supposent d'ouvrir la boite noire des techniques pour y dénouer les fils des pratiques sociales qui leur donnent tout leur sens. Ni opposition, ni mise en service d'une partie par l'autre, la recherche technologique implique la mise en place d'un dialogue animé par les enjeux mêmes de chaque domaine de recherche. 
Les produits, services et systèmes techniques ne résultent pas de la simple application d'une science qui les précéderait. Si l'on se plaçait dans un tel cadre, les technologies cognitives par exemple, devraient être des «sciences cognitives appliquées ». Or, bien que les sciences cognitives soient encore loin d'offrir quelques résultats définitifs, nous sommes depuis longtemps entourés d'une infinité de supports techniques pour toutes nos activités cognitives de perception, de mémorisation, d'orientation spatiale et temporelle, de déduction, d'imagination, d'interaction, etc. Jack Goody a défendu que depuis l'origine de l'écriture, les techniques graphiques de la formule, de la liste et du tableau, ne sont pas simplement des modes de spatialisation des connaissances, mais que par leurs structures, elles participent à la production de nouveaux contenus, et de nouvelles opérations cognitives, ce qu'on appelle la « raison graphique » (Goody, 1979). L'écriture précède la grammaire, comme la longue vue précède la dioptrique, ou les systèmes d'échange précèdent la science économique (Jorion, 1994). Dès lors, il est nécessaire de renverser l'approche applicative pour plutôt d'abord considérer le fait technique comme l'objet possible d'une recherche fondamentale sur sa nature et ses conditions, sur ses effets comme sur sa dynamique historique et sociale, questions qui prennent toute leur importance à notre époque de la technoscience et de l'innovation accélérée. Cette « avance de la technique » résulte de son caractère constitutif (Stiegler, 1994). C'est la raison intrinsèque d'une recherche fondamentale (et non pas applicative) sur la technique.

Il nous semble qu'en montrant la complexité des interactions entre développements techniques, usages, et activités sociales, les disciplines des sciences humaines ne doivent en rien abandonner la rigueur de leur questionnement intrinsèque, de leurs méthodes et de leur histoire propre. Cependant, en posant la question du fait technique au cœur de leurs recherches, elles se mettent en position de renouveler leurs problématiques fondamentales. En effet, la question de la constitutivité technique impacte aussi bien leurs théories, que leurs fondements paradigmatiques et leurs méthodes.

Prenons un exemple dans le champ des sciences cognitives. L'échec relatif de l'Intelligence Artificielle est typique de l'échec de l'ambition de réaliser un développement technique par l'application d'une théorie de la cognition (qui était celle de la computation interne de représentations formelles). Mais ayant échoué à faire des « machines qui pensent » ce domaine de recherche à rencontré un immense succès dans le développement de «machines qui aident à penser » (Bachimont, 1994). En effet, la structure combinatoire de l'information numérique a démontré la puissance d'une structure en composant des symboles formels pour le pointage quasi directe dans de gigantesque bases de données (extraordinaire efficacité des moteurs de recherche), pour l'organisation des connaissances (ontologies), pour le traitement automatique du langage naturel (traitement de textes, aide à la traduction), le travail coopératif (réseaux, Computer-Supported Cooperative Work), et maintenant l'organisation des réseaux sociaux.

Cet apparent paradoxe d'un échec scientifique associé à un succès technologique se comprend si l'on reconnait que le cognitivisme classique a oublié que le paradigme computationnel sur lequel il se fondait avait été justement construit sur le modèle de la technique de l'écriture. C'est la technique d'écriture et lecture de symboles mathématique qui avait servi à Turing (suivant en cela le programme de Hilbert) comme modèle pour concevoir la machine virtuelle dont l'ordinateur est une réalisation matérielle. Ordinateur qui, comme système de traitement d'une information indépendante de son support matériel devait servir de modèle à l'approche fonctionnaliste du cognitivisme classique (Gardner, 1987 ; Haugeland, 1989). Là encore, la technique précède le champ théorique qu'elle rend possible. On comprend mieux à la fois l'échec du programme fort de l'Intelligence Artificielle et le succès des technologies qui s'inscrivent dans le paradigme computationnel. Ils correspondent à une pertinence technique, et non pas de sciences cognitives.

Mais, du coup, ce sont les sciences cognitives qui se trouvent renouvelées. Plutôt que de prendre le modèle d'un fonctionnement technique (l'écriture mathématique et la machine de Turing) pour rendre compte d'une cognition supposée «naturelle», il s'agit maintenant de rendre compte du fonctionnement de cette technicité essentielle de la cognition. Comme le remarque avec humour Bruno Latour, ce que 
nous apprend l'intelligence artificielle c'est que depuis toujours l'intelligence est artificielle (Latour, 1992). Dès lors, charge aux sciences cognitives de rendre compte de la cognition comme outillée, ce qui conduit à une approche externaliste telle qu'on le trouve dans le paradigme alternatif de la cognition incarnée, située ou distribuée (embodied and embedded) (Chemero, 2009 ; Lenay \& Steiner, 2010 ; De Preester, 2011). Plutôt que par des représentations mentales internes, l'activité cognitive se déroulerait dans un environnement outillé qui s'étend dans l'espace même des inscriptions externes. Ce champ de recherche se développe actuellement sous le titre de «l'extended mind theory » (Clark \& Chalmers, 1998). La cognition n'est plus désincarnée, indépendante de son support matériel. Dans ce cadre, l'approche énactive se fonde sur l'inscription corporelle de la cognition en cherchant aussi à comprendre comment les supports techniques participent à l'énaction de l'expérience vécue et des opérations cognitives (Varela et al., 1999).

\section{Renouvellement du dialogue interdisciplinaire}

La question de la constitutivité technique n'est pas réservée à une discipline particulière, comme par exemple l'ergonomie, les IHM (Interfaces Homme Machine), ou le design, mais concerne chacune des disciplines des SHS. En effet, s'il ne s'agissait que d'un domaine de spécialité on rencontrerait le danger de prendre simplement comme acquis les savoirs de disciplines plus générales et fondamentales comme par exemple la psychologie, la sociologie, l'économie, alors que, au contraire, il s'agit de comprendre la constitutivité technique de ces disciplines elles-mêmes et de leurs objets.

Ce renversement de point de vue a une grande importance pour le dialogue interdisciplinaire. En effet, il s'agit d'imposer des contraintes aux explications des disciplines partenaires et non pas seulement en recevoir. Par exemple, pour rester dans le domaine des sciences cognitives, plutôt que de travailler à la conception d'interfaces homme-machine en prenant les résultats des neurosciences comme des savoirs externes stabilisés, il s'agit au contraire, partant de l'étude de la façon dont des figures ou des symboles inscrits sur des supports matériels sont constitutifs d'opérations cognitives majeures comme la catégorisation ou l'idéation, de revenir vers les neuroscience et de les questionner sur les phénomènes de plasticité cérébrale susceptibles de rendre compte de la façon dont l'organisme peut s'approprier les outils qu'il saisi, comment il peut participer à l'interprétation des traces qu'il produit, comment il peut apprendre les stratégies qui conviennent à la formation d'une cognition étendue dans l'espace.

La réalité des terrains d'étude ou des réalisations force déjà par elle-même la multiplicité des points de vue disciplinaires. Mais si l'on veut qu'elle participe de recherches proprement scientifiques, il faut que cette rencontre ne s'effectue pas seulement aux frontières des disciplines, mais bien aussi par le cœur des questions scientifiques que chacune approfondit.

Travailler à tous les niveaux de complexité sur la relation entre dispositifs techniques, connaissances et dynamique sociale, implique une réflexivité puisque, si les supports et agencements sont constitutifs des connaissances, cela concerne en premier lieu les activités scientifiques, philosophiques ou historiques: supports numériques d'écriture et de lecture, de traitement de données, d'organisation collaborative via les réseaux, de diffusion, de valorisation, de propriété intellectuelle, etc. Prenant au sérieux les conditions techniques de la constitution des connaissances, on peut suivre le modèle de la critique philosophique proposée par la déconstruction derridienne : la technique de l'écriture comme condition constitutive de la philosophie qui réalise cette critique de l'écriture (Derrida, 1962 ; Sebbah, 2008).

Or, cette réflexivité est croisée. Les conditions concrètes du travail de chaque discipline se trouvent être l'objet de recherches possibles pour les autres disciplines, et réciproquement. Pour décrire le travail interdisciplinaire, on utilise souvent les termes « croisement des disciplines ». Pour notre part, nous prenons au sérieux cette expression en l'entendant sur le modèle du croisement de regard. En effet, dans cette rencontre perceptive, chacun est compris dans le regard de l'autre et est justement vu pour ce qu'il ne voit pas lui-même puisque cela lui sert à voir, ici ses yeux, qui dans cette métaphore représentent 
l'incarnation des moyens, méthodes et principes théoriques de chaque perspective disciplinaire. Ainsi les différentes disciplines, plutôt que de s'installer dans l'indifférence réciproque, interagissent en questionnant mutuellement leurs présupposés techniques, méthodologiques et théoriques.

Par exemple, alors que le sociologue des sciences étudie la vie de laboratoire pour montrer comment le psychologue construit ses expériences, les recherches menées dans ce laboratoire concernent justement la façon dont la spatialisation de l'information par l'écriture participe à la constitution des catégories et des opérations cognitives, ce qui en retour conduit à une analyse critique des outils de la sociologie, comme les questionnaires, la construction de tableaux de données, ou l'interprétation des cartographies de réseaux (Bruno Latour, 1987).

\section{Retour sur la formation}

Cette approche pour une recherche technologique en SHS est maintenant portée par le GIS que nous mettons en place. Elle s'inscrit dans le contexte des écoles d'ingénieur et impacte directement leur formation. Elle montre bien que la formation en sciences humaines et sociales ne peut pas simplement viser à «l'adaptation des profils des ingénieurs à un environnement socio-économique prédéfini ». Cette formation doit bien plutôt offrir des outils conceptuels et des méthodes qui puissent les aider, comme technologues et comme citoyens, à participer à la construction d'un environnement socio-économique en devenir. Et pour cela, elle doit introduire les étudiants à une dynamique de recherche en SHS (et non pas délivrer des savoirs prétendument achevés). Les métiers et les modes d'intervention des ingénieurs subissent des évolutions rapides, variées, essentiellement imprévisibles. Ils sont de plus en plus amenés à concevoir des situations d'activités plus que des dispositifs techniques. Ces situations intègrent des solutions techniques mais aussi des prescriptions organisationnelles et sociétales (explicites ou implicites), des schémas d'usage, voire une idéologie particulière de la technique (allocation des rôles entre l'homme et la machine par exemple). Ces situations ont la particularité de s'étendre dans le temps et dans l'espace (physique et social) et nécessitent des solutions "intégrées » et négociées entre de multiples acteurs humains ou non-humains (Latour, 1999). Les compétences transmises en SHS aux élèves-ingénieurs doivent être pensées comme une introduction à une démarche de recherche qui les prépare à imaginer et concevoir des situations à venir inconnues et qui leur permette d'acquérir une posture critique dans la constitution des nouveaux objets socio-techniques. L'intervention de l'ingénieur dans le monde industriel s'inscrit donc clairement dans une recherche technologique qui concerne aussi bien les sciences humaines que les sciences de l'ingénieur (Choplin, 2013). Une telle recherche en SHS propose des méthodes qui peuvent participer directement aux processus de conception euxmêmes, dans l'esprit de la réflexion thématisée notamment par Herbert Simon autour des « sciences de l'artificiel »; en aidant à comprendre le sens des techniques, pour agir, imaginer, créer, dialoguer et intervenir socialement (Simon, 1969, 1991). On observe d'ailleurs actuellement une évolution depuis la conception centrée sur l'utilisateur, puis sur les usages ou services, vers la conception comme constitution d'une expérience vécue ${ }^{12}$.

De même, il n'y a pas à proprement parler «d'acceptabilité sociale du changement technique », mais le plus souvent une co-construction, co-conception, plus ou moins heureuse, réussie ou non, dans un réseau d'acteurs qui échangent sans cesse leurs rôles de décideurs, concepteurs, utilisateurs ou producteurs. On ne peut donc restreindre l'apport des sciences humaines et sociales sur la technologie à un habillage « humaniste » visant à assurer l'acceptabilité sociale de la technologie. Ce lieu commun vient d'un temps où processus de conception, de fabrication et de consommation pouvaient être séquentiellement départagés. Au contraire, la recherche peut viser à comprendre et à définir les dispositifs socio-techniques situés historiquement et socialement. En effet, l'innovation, vue comme

\footnotetext{
12 Par exemple une spécialité de master en UxD (User eXperience Design) a été mise en place à l’UTC en 2012.
} 
changement technique ou sociotechnique, qu'elle soit incrémentale ou de rupture, n'est pas une donnée en soi. Les processus d'innovation sont construits et sont l'aboutissement d'interactions complexes entre acteurs et dispositifs au sein de ces réseaux sociotechniques (Callon, 2002 ; Von Hippel, 2007, 2009) Les rôles du milieu et de la disponibilité en ressources de médiation, les modalités des phénomènes d'apprentissage à l'œuvre, sont autant d'axes potentiels de recherche. Ces questions sont au cœur d'une meilleure compréhension des objets sociotechniques innovants. La recherche en SHS « sur la conception », c'est-à-dire sur les processus et méthode de l'invention et de la diffusion, peut ainsi évoluer vers une recherche en SHS « de conception », c'est-à-dire une recherche qui participe aux réseaux de conception eux-mêmes et s'enrichit de l'apport de ces réseaux (Le Masson, Weil, \& Hatchuel, 2006 ; Forest, Méhier, \& Micaëlli, 2005). Dès lors, les catégories d'activités d'ingénierie de conceptions innovantes comme la R\&D ou le «transfert technologique », considérées souvent comme des catégories «naturelles », apparaitront ainsi pour ce qu'elles sont, des élaborations conceptuelles construites et qui trouvent corps à un moment donné dans certains modèles d'organisation. Cette recherche en SHS prendrait ainsi toute sa place au cœur de l'ingénierie de conception, et notamment dans l'élaboration des modèles conceptuels comme dans leur implémentation organisationnelle concrète.

\section{Conclusion}

On a écrit que l'instrumentalisation des disciplines de sciences humaines, provoquant une perte d'autonomie de ce secteur de recherche, déboucherait sur un rôle inefficace de ces disciplines, et une perte de leur valeur scientifique puisque les objets de la recherche ne relèveraient pas d'enjeux internes aux développements et débats qui animent la discipline. Dans l'approche proposée ici, il ne s'agit pas de faire disparaitre les différences disciplinaires, ni même de créer une discipline nouvelle, mais de constituer un travail d'équipe interdisciplinaire qui intègre en son sein les sciences pour l'ingénieur suivant les objets étudiés. Un dialogue constructif et essentiel dans les écoles d'ingénieurs qui est donc aussi particulièrement urgent pour la compréhension des mutations rapides du monde contemporain. A condition de considérer en amont le sens social des choix techniques, et pas seulement, comme c'est encore souvent le cas, ne considérer que les conséquences sociales en aval d'innovations techniques réalisées d'abord dans l'opacité et l'ignorance. Au delà de l'opposition entre sciences de la nature et sciences de l'homme, ceci permettrait de faire fructifier les sciences de l'artificiel (Simon, 1969).

Il semble possible de se donner les outils et environnements nécessaires pour constituer une telle unité autour des technologies des SPI et des SHS et initier une véritable réflexion sur la nature nécessairement interdisciplinaire du projet de l'ingénierie (Moriarty, 2008). C'est l'objet du GIS UTSH que nous sommes en train de mettre en place avec les équipes de SHS inscrites dans le réseau des universités de technologie. Mais ceci ne constitue de notre point de vue qu'une étape : l'objectif est d'étendre cette initiative à l'ensemble des écoles d'ingénieurs concernées par cette problématique et qui y trouveront un écho à leurs préoccupations locales.

On peut également espérer que cette démarche pourra être relayée par les structures institutionnelles d'organisation et de pilotage des établissements nationaux dispensant une formation en ingénierie (voir par exemple quelques initiatives récentes prises par la Conférence des Directeurs des Ecoles Françaises d'Ingénieurs - CDFI).

\section{Références}

Akrich, M. (1987). Comment décrire les objets techniques ? Techniques \& Culture, 9, 4964.

Akrich, M. (1994). Comment sortir de la dichotomie technique/société. In B. Latour \& P. Lemonnier (Éd.), De la préhistoire aux missiles balistiques : l'intelligence sociale des techniques (p. 105-131). Paris : La Découverte. 
Les sciences humaines dans les parcours scientifiques et techniques professionnalisants

Auroux, S. (1990). La révolution technologique de la grammatisation : introduction à l'histoire des sciences du langage. Paris : Gallimard.

Bachimont, B. (1994). Artéfacture : des machines qui pensent aux machines qui donnent à penser. Techniques \& culture, (23-24), 275-306.

Becker, A., \& Bloch, É. (2006). L’histoire, la guerre, la résistance. Gallimard.

Bernard, M.-Y. (1970). Les Instituts universitaires de technologie. Paris : Dunod.

Bloch, M. (1957). L'étrange défaite (Folio.). Paris : Gallimard.

Bodé, G. (2002). L'enseignement technique de la Révolution à nos jours (I NRP., Vol. 2 tomes). Paris.

Boirel, R. (1983). L'avènement de l'INSA.- Lyon : Association des anciens élèves de l'INSA (p. 171).

Bonnet, B., \& Terral, H. (2011). La formation des maîtres de CAP aux lendemains de la Libération ? : l'enjeu des «humanités techniques » (p. 16 p.). Présenté à Le centenaire du CAP. Consulté à l'adresse http://gresco.labo.univ-poitiers.fr/sites/gresco.labo.univ-poitiers.fr/IMG/ pdf/BONNET-TERRAL-2.pdf

Callon, M. (2002). From science as an economic activity to socioeconomics of scientific research. The dynamics of emergent and consolidated techno-economic networks. In P. Mirowski \& E.-M. Sent (Éd.), Science Bought and Sold. Essays in the Economics of Science (p. 277-317). Chicago and London : The University of Chicago Press.

Charlot, B., \& Figeat, M. (1985). Histoire de la formation des ouvriers. 1789-1984. Paris : Minerve.

Charmasson, T. (Éd.). (1987). L'Enseignement technique de la Révolution à nos jours. Textes officiels avec introduction, notes et annexes (INRP et Économica.). Paris.

Chemero, A. (2009). Radical embodied cognitive science. The MIT Press.

Choplin, H. (2013). L'ingénieur contemporain, le philosophe et le scientifique. Paris : Encre marine / Belles Lettres.

Clark, A., \& Chalmers, D. (1998). The extended mind. Analysis, 58(1), 7-19.

Cresswell, R. (2011). Techniques et culture : les bases d'un programme de travail. Techniques \& culture, 191(1), 21-45.

De Preester, H. (2011). Technology and the Body : the (Im) Possibilities of Re-embodiment. Foundations of Science, 1-19.

Derrida, J. (1962). Introduction à «L'Origine de la géométrie » de Husserl. Paris : PUF.

Forest, J., Méhier, C., \& Micaëlli, J.-P. (2005). Pour une science de la conception. Fondements, méthodes, pratiques. Belfort : UTBM.

Garçon, A.-F. (2012). L'Imaginaire et la pensée technique - Une approches historiques, XVIe-XXe siècle. Paris : Classiques Garnier.

Gardner, H. (1987). The mind's new science : A history of the cognitive revolution. Basic books.

Goody, J. (1979). La raison graphique. Editions de minuit Paris.

Haudricourt, A.-G. (1988). La technologie, science humaine : recherches d'histoire et d'ethnologie des techniques. Les Editions de la MSH.

Haudricourt, A.-G. (2011). Des gestes aux techniques. Essai sur les techniques dans les sociétés prémachinistes. (J.-F. Bert, Éd.) (Editions Quae.). Versailles - Paris : Maison des sciences de l'homme.

Haugeland, J. (1989). L'esprit dans la machine. Odile Jacob.

Jeneveau, A. (2011). Les sciences humaines et sociales dans la formation des ingénieurs : enjeux, repères, questions et perspectives. In Les sciences humaines et sociales dans les écoles d'ingénieurs (p. 1722). Consulté à l'adresse http://crf.cnam.fr/medias/fichier/actes-coloque-: ingenium-dec-2011_1330013578134.pdf

Jorion, P. (1994). La queue qui remue le chien : métamorphose de la finance due à son informatisation. Techniques \& culture, (23-24), 307-349.

Lamard, P., \& Lequin, Y.-C. (2012a). Entre Villetaneuse et Compiègne (1944-1972). L'université de sciences et techniques Paris-Nord. In J. Girault, J.-C. Lescure, \& L. Vadelorge (Éd.), Paris XIII histoire d'une université en banlieue (1970-2010) (p. 198215). Paris : Berg international éditeurs. 
Lamard, P., \& Lequin, Y.-C. (2012b). Universités nouvelles, technique et technologie (France : 19682010). In L. Roy \& Y. Gingras (Éd.), Les universités nouvelles, enjeux et perspectives (p. 110138). Presses de l'Université du Québec.

Lamard, P., \& Lequin, Y.-C. (2012c). Guy Deniélou (1923-2008), une certaine idée de la technologie. In D. Parrochia \& V. Tirloni (Éd.), Formes, systèmes et milieux techniques après Simondon (p. 7182). Jacques André Editeur.

Lamard, Pierre, \& Lequin, Y.-C. (2005). La technologie entre à l'université. Compiègne, Sevenans, Belfort-Montbéliard, Belfort-Montbéliard, UTBM. Consulté à l'adresse http://www . lavoisier.fr/livre/notice.asp?ouvrage $=2263207$

Laot, F. (1999). La formation des adultes : Histoire d'une utopie en acte-Le complexe de Nancy. Paris : L'Harmattan.

Latour, B. (1992). Des quelques services rendus par l'IA à la philosophie des sciences. In C. Sayettat (Éd.), l'Intelligence Artificielle, une discipline et un carrefour interdisciplinaire (p. 55-65). Compiègne.

Latour, B. (1999). Politiques de la nature. Comment faire entrer les sciences en démocratie. $L a$ découverte.

Latour, Bruno. (1987). Les «vues » de l'esprit. Réseaux, 5(27), 79-96.

Le Masson, P., Weil, B., \& Hatchuel, A. (2006). Les processus d'innovation : Conception innovante et croissance des entreprises. Paris : Hermes science publication. Consulté à l'adresse http://rfg.revuesonline.com/gratuit/RFG33_175_14_Actua-livres.pdf

Lemonnier, P. (1983). L'étude des systèmes techniques, une urgence en technologie culturelle. Techniques \& Culture. Revue semestrielle d'anthropologie des techniques, (1).

Lenay, C., \& Steiner, P. (2010). Beyond the internalism/externalism debate : the constitution of the space of perception. Consciousness and Cognition (19), 938-952.

Léon, A. (1968). Histoire de l'éducation technique (Vol. 938). Presses universitaires de France.

Lequin, P., \& Lamard, P. (2011). Compiègne, 1972 : L'université de technologie est-elle enfant de 1968 ? In Réformer l'Université après mai 68. La loi d'orientation de l'enseignement supérieur et son application (1968-1984). Présenté à Colloque Fondation Charles de Gaulle, Paris.

Lequin, Y.-C. (2009). La technologie est une science humaine. Sciences Humaines, 205, 24-29.

Lequin, Y.-C. (2013). L'INSA de Lyon. Origines, contexte et portée. Note pour l'équipe Geoscience $(A N R)$ (p. 19).

Leroi-Gourhan, A. (1964). Le geste et la parole : technique et langage (Vol. 1). Albin Michel Paris.

Longchambon, H. (1958). Les Sciences sociales en France. Un bilan, un programme. In Annales. Histoire, Sciences Sociales (Vol. 13, p. 94-109).

Mauss, M. (1970). Techniques, technologie et civilisation. (N. Schlanger, Éd.) (Quadrige.). PUF.

Mialaret, G. (1997). Plan Langevin-Wallon. Paris : PUF.

Mitcham, C. (1994). Thinking through technology: The path between engineering and philosophy. Chicago : University of Chicago Press.

Moriarty, G. (2008). Engineering Project : Its Nature, Ethics, and Promise. University Park, PA : Penn State Press.

Musselin, C. (2001). La longue marche des universités françaises. Paris : Presses Universitaires de France.

Prost, A. (1997). Mort et naissance de l'université française. In Education, société et politique. Une histoire de l'enseignement de 1945 à nos jours (p. 133155). Paris : Seuil.

Schlanger, N. (2012). Une technologie engagée. In Mauss M. Techniques, technologie et civilisation (p. 17134). Paris : PUF.

Schmid, A.-F., Mambrini-Doudet, M., \& Hatchuel, A. (2011). Une nouvelle logique de l'interdisciplinarité. Nouvelles perspectives en sciences sociales : Revue internationale de systémique complexe et d'études relationnelles, 7(1), 105-136. 
Sebbah, F. D. (2008). Ecriture et Invention chez Derrida.

Sebbah, F. D. (2010). Qu'est-ce que la technoscience? Une thèse épistémologique ou la fille du diable? Paris : Encre Marine/Les Belles Lettres.

Sigaut, F. (2002). La formule de Mauss. Techniques \& culture, (40), 153-168.

Sigaut, F. (2010). Postface. In Haudricourt, A.-G. Des gestes aux techniques. Essai sur les techniques dans les sociétés pré-machinistes (Editions de la Maison des Sciences de l'Homme., p. 217-224). Paris : Editions Quae.

Simon, H. A. (1969). The sciences of the artificial. Cambridge, MA.

Simon, H. A. (1991). Sciences des systèmes, sciences de l'artificiel. (J.-L. Le Moigne, Trad.). Dunod.

Steiner, P. (2010). Philosophie, technologie et cognition W : quelques enjeux. Intellectica, (53/54), 740.

Stiegler, B. (1994). La technique et le temps, tome 1. La Faute d'Epiméthée, Galilée.

Varela, F. J., Thompson, E., \& Rosch, E. (1999). The embodied mind : Cognitive science and human experience. MIT press.

Von Hippel, E. (2007). The sources of innovation. Springer.

Von Hippel, E. (2009). Democratizing innovation : the evolving phenomenon of user innovation. International Journal of Innovation Science, 1(1), 29-40. 